\title{
On subsonic near-wake flows of a space launcher configuration with various base geometries
}

\author{
Dominik Saile $^{1} \mathbb{D} \cdot$ Viktor Kühl $^{1} \cdot$ Ali Gülhan $^{1}$
}

Received: 30 August 2019 / Revised: 20 October 2020 / Accepted: 16 January 2021 / Published online: 19 May 2021

(c) The Author(s) 2021

\begin{abstract}
Buffet/buffeting as load imposing mechanism on the base structures of space launcher has been of strong interest ever since it was found as partially responsible for the failed flight 157 of Ariane 5. Several studies suggested that the base region is most excited at Mach 0.8. A preceding study of the current series on base flow effects revealed a differing excitation in comparison to the other subsonic Mach number cases. It featured an especially pronounced excitation in the recirculation region. Thus, the current work attempts to answer the question why this case appears to be distinct. This is done by decreasing the relative nozzle length and focusing on the Reynolds stress distribution. The research question is approached by experiments in the 'Vertical Test Section Cologne' (VMK) on a base model with supersonic, over-expanded exhaust jet exposed to an ambient flow at Mach 0.8 and a Reynolds number of $1.4 \cdot 10^{6}$. Data are acquired by means of particle image velocimetry (PIV) and high-speed schlieren imaging. The results reveal that a most unfavorable configuration appears to exist, which is if the mean shear layer reattachment takes place just on the tip of the nozzle.
\end{abstract}

\section{Graphic abstract}


Extended author information available on the last page of the article 


\section{Introduction}

The failure of Ariane 5 during flight 157 was partially attributed to the buffet/buffeting effect. Here, buffet refers to the excitation of aerodynamic forces in the wake and buffeting the structural response. In resonance, the interaction between aerodynamic and structural loads can have disastrous consequences. The studies of David and Radulovic (2005), Schwane (2015) have found that flow conditions are especially detrimental for Ariane 5 in the transonic flow regime, in particular, at Mach 0.8.

The failed flight has triggered many studies to improve and extend the understanding of base flow phenomena. Investigations on similar base geometry representations for Ariane 5 have been conducted by Wong et al. (2007), Schrijer et al. (2011), Hannemann et al. (2011), Schwane (2015). On generic configurations, the governing mechanisms for buffeting have been analyzed among others by Fuchs et al. (1979), Deprés et al. (2004), Deck and Thorigny (2007), Weiss et al. (2009), Weiss and Deck (2013), Schrijer et al. (2014), Scharnowski (2013), Scharnowski et al. (2015), Scharnowski et al. (2016), Statnikov et al. (2016), Statnikov et al. (2017), van Gent et al. (2017b), and van Gent et al. (2017a). Statnikov et al. (2017) extended existing knowledge about governing mechanisms by means of 'dynamic mode decomposition' and isolated three different modes of excitation in the base region: 'cross-pumping' of the separation bubble and 'cross-flapping' and 'swinging' of the shear layer.

Few of the references above are experimental and even fewer with a supersonic exhaust jet (Deprés et al. 2004; Weiss and Deck 2013; van Gent et al. 2017b, 2019), meaning there is generally a lack experimental data. However, van Gent et al. (2017b), van Gent et al. (2019) actually conducted extensive work similar to the study at hand, meaning these results can be applied for validation purposes and comparisons. In that study, the impact of the nozzle length was studied for an ambient flow in the transonic regime and in the supersonic regime. For the transonic case, it was found that for comparable overall conditions (geometry+flow conditions) 'neither an increase in nozzle length nor the presence of an exhaust plume' lead to 'a significant change in the mean reattachment length.' Further, it was concluded that the plume 'cannot accurately be modeled by replacing the plume with a solid geometry.'

The first statement cannot be generally supported by the predecessor studies of the current work. A comparison between the jet-off- (Saile et al. 2019b) and jet-on-case (Saile et al. 2019a) shows clear differences when the jet is turned on. It decreased from $L_{r} / D=1.18$ to 0.94 , which corresponds to a retraction by $20 \%$ (Saile 2019). There, this was attributed to a base suction effect as previously described by Schoones and Bannink (1998), Deprés et al. (2004); Wolf (2013).

However, the second statement though can be supported. According van Gent et al. (2017b), van Gent et al. (2019), the plume cannot be correctly modeled with a solid plumelike sting since, on the one hand, the shape of the plume is not known a priori, and on the other hand, since the jet accelerates the outer flow due to entrainment. For the work at hand, a third aspect comes into play which concerns a presumable feedback mechanism with the jet leading to a strong excitation.

The feedback taking place in the near-wake region was suggested in Saile et al. (2019a); Saile (2019), and the excitation in question can be found in particular for Mach 0.8 when the jet is turned on. It is found that the excitation is outstanding in comparison to other investigated subsonic ambient Mach numbers (Saile et al. 2019a, b), which provides the motivation for the current study. Thus, the following questions are addressed:

- How does this outstanding excitation react to a nozzle length change?

- Is it possible to identify a favorable or worst-case configuration dependent on the relative nozzle length?

- Is it possible to identify particularities leading to this outstanding excitation?

Thus, the objective here is to study the impact of the relative nozzle length on the flow field of a simplified space launcher configuration. More generally, among other studies such as the ones noted above, the overarching idea here is to provide data to the community as a small contribution for the development of further design guidelines for space launchers such as asked in the three bullet points above.

As in the previous studies of this series (Saile et al. 2019a, b), this task is approached by conducting experiments in the 'Vertical Test Section Cologne' (VMK) on a base model representing generic space launcher configurations. The base model geometry is modified from test to test by changing the nozzle length. Experiments are executed at Mach 0.8 for a Reynolds number of $1.4 \cdot 10^{6}$. Data are captured by means of particle image velocimetry (PIV), and the results mainly concern the mean and turbulent quantities for the velocity. A POD analysis is applied to extract dominant velocity modes. To underline some points over the course of the discussion, the probability density estimate function (PDF) derived by applying a kernel smoothing density (ksd) function is given of a point just upstream of the nozzle exit and selected instantaneous velocity distributions are shown. Additionally, the dynamic mechanisms of the near-wake are captured with high-speed schlieren images and the results are analyzed with respect to the spectral content. 
The paper is structured as following: Hereafter in Sect. 2, the methods for data acquisition and analysis are presented followed by the description of the results in Sect. 3. A conclusion and outlook is given in Sect. 4.

\section{Methods}

The experiments were conducted in VMK (Triesch and Krohn 1986; DLR 2019; Saile et al. 2015), which is a blowdown type wind tunnel with a free test section. The current setup is comparable to the experiments presented in detail in Saile et al. (2019b) using again the subsonic wind tunnel nozzle with an exit diameter of $340 \mathrm{~mm}$ into which the wind tunnel model is integrated (Fig. 1). The main components mimic generically the base geometry of Ariane 5, and for completeness, and its details are presented again in Fig. 2 . This baseline model is modified by attaching adapters of various lengths to the base enabling measurements with a relative nozzle length of $L / D=0.45,0.6,0.75,0.9,1.05$, and 1.20 (similar to the approach in Saile et al. (2013), van Gent et al. (2017b)). The nozzle remains the same for all base geometries. It has a conical contour, and to avoid the occurrence of condensed oxygen in the cold exhaust jet, it was chosen to limit the expansion ratio to $\epsilon=7.37$ (nozzle exit Mach number of 3.59 for air).

The ambient flow Mach number is kept at 0.8 for all configurations and further details to the flow conditions are

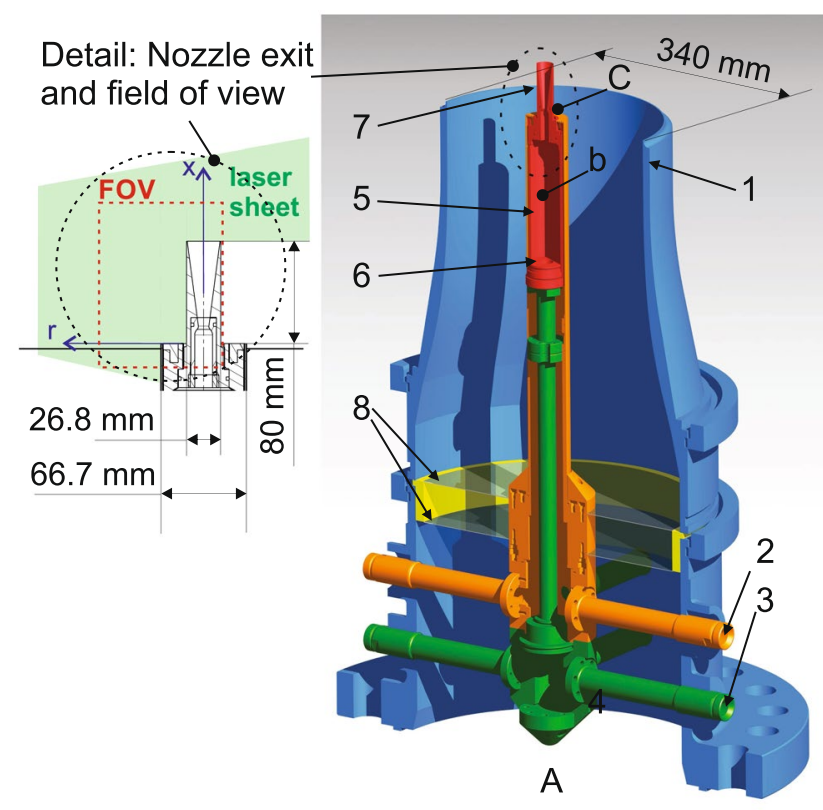

Fig. 1 Sketch of the wind tunnel model (introduced first in (Saile et al. 2019b)) with the wind tunnel nozzle (blue), cold jet supply system (green) and the chamber (red). The graph further shows the field of view $(F O V)$

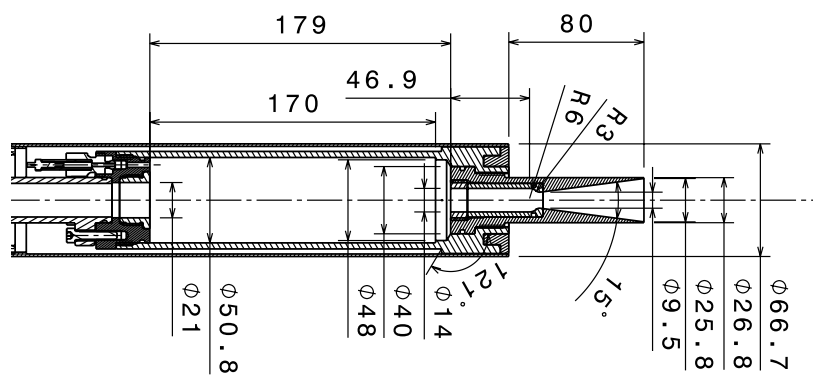

Fig. 2 Technical drawing focusing on the chamber and nozzle geometry (introduced first in (Saile et al. 2019a)). Units are given in millimeter

listed in Table 1. It contains the exit Mach number $M a_{C}$, exit velocity $U_{C}$, exit Reynolds number $R e_{C}$ based on the diameter of the main cylinder. Additionally, the chamber pressure $p_{0, b}$ and temperature $T_{0, b}$ of the wind tunnel model are listed. The exit Mach number was calculated under the assumption of an isentropic expansion by means of the reservoir and ambient pressure; the velocity is directly taken from the PIV results. Due to corrupted results just upstream in the vicinity of the base separation point, the ambient flow (Table 1) was determined farther downstream from the base. The ambient flow is averaged over an area between $0.15<x / D<0.2$ and $0.7<r / D<0.8$.

The preceding studies additionally provide data of the incoming boundary layer for Mach 0.8 and a relative nozzle length of $L / D=1.2$. Since only adapters have been added to the base of the model, it is assumed that the changes regarding the incoming boundary layer are negligible. For more details, please refer to Ref. (Saile et al. 2019a, b).

The current experimental settings seek to simulate the conditions of Ariane 5 at an altitude of $4.3 \mathrm{~km}$. This altitude is equivalent to an ambient Mach number of 0.8 , which is correspondingly kept similar between the real-flight configuration and the experiments. Due to the sheer size of Ariane 5, it is not possible to establish a diameter-based Reynolds number similarity $\left(70 \cdot 10^{6}\right.$ vs. $1.4 \cdot 10^{6}$ for the tests at hand). However, the near-wake flow of both configurations is turbulent and can be attributed to the trans-or post-critical regime Morkovin (1964) capturing similar fluid dynamic effects. Further, the incoming boundary layer is thin with respect to the step height (from the main cylinder to the nozzle). For such flows, Roshko and Lau (1965) and Westphal et al. (1984) found the recirculation region to be similar.

Nevertheless, due to the geometric differences and due to the usage of cold air to simulate the exhaust jet, the corresponding flow similarity can only be partial. For instance, similarity can be established with respect to the shape of the plume: an over-expanded nozzle flow is present for both conditions. Accordingly, the pressure ratio between the ambient flow and jet agrees with a reasonable degree, meaning 
Table 1 Freestream conditions and reservoir conditions of the wind tunnel model

\begin{tabular}{lllllll}
\hline $\begin{array}{l}L / D \\
{[-]}\end{array}$ & $\begin{array}{l}M a_{C} \\
{[-]}\end{array}$ & $\begin{array}{l}U_{C} \\
{\left[\mathrm{~ms}^{-1}\right]}\end{array}$ & $\begin{array}{l}R_{C} \\
{[-]}\end{array}$ & $\begin{array}{l}p_{0, b} \\
{[\mathrm{MPa}]}\end{array}$ & $\begin{array}{l}T_{0, b} \\
{[K]}\end{array}$ & $\begin{array}{l}{\left[x_{v c}, r_{v c}\right] / D} \\
{[-]}\end{array}$ \\
\hline 1.2 & 0.79 & 257.5 & $1.4 \cdot 10^{6}$ & 3.31 & 286.1 & {$[0.48,0.35]$} \\
1.05 & 0.80 & 263.4 & $1.4 \cdot 10^{6}$ & 3.22 & 287.7 & {$[0.58,0.36]$} \\
0.9 & 0.79 & 261.5 & $1.4 \cdot 10^{6}$ & 3.25 & 289.2 & {$[0.47,0.36]$} \\
0.75 & 0.80 & 261.6 & $1.4 \cdot 10^{6}$ & 3.23 & 286.5 & {$[0.5,0.36]$} \\
0.6 & 0.79 & 265.1 & $1.4 \cdot 10^{6}$ & 3.23 & 282.4 & {$[0.56,0.36]$} \\
0.45 & 0.79 & 265.7 & $1.4 \cdot 10^{6}$ & 3.22 & 287.2 & {$[0.53,0.36]$} \\
\hline
\end{tabular}

a ratio of 0.25 can be found for Ariane 5 vs. of 0.36 for the experiments. In other words, the displacement effect can be considered as similar.

The largest deviation without doubt is induced by the conditions in the combustion chamber/reservoir. To compensate for the difference regarding the specific heats $\kappa$, which influences the pressure change caused by a change in the flow direction for a supersonic flow, Goethert and Barnes (1960) suggested to keep $\kappa M a_{j}^{2} / \sqrt{M a_{j}^{2}-1}$ similar. With 5.2 vs. 5.6 for Ariane 5, this quantity agrees quite well. Note that $M a_{j}$ corresponds to the jet Mach number. Next, the difference concerning the chamber temperatures is addressed, which directly influences the jet velocity. The jet velocity is about six to seven times larger for Ariane 5. Interrelated to that is the base suction effect. Thus, it must be assumed that shear layer evolution defers and the overall length of the mean recirculation bubble is presumably underestimated. Note that the Ariane 5-related flight data are based on a system analysis executed and provided by the DLR Institute of Space Systems. Further details in that respect and with respect to the similarity considerations are presented in Saile (2019).

The PIV measurement setup features a minor difference regarding the field of view (FOV), but other than that, the setup was kept just as in Saile et al. (2019a), meaning a classical 2D-2C setup was used. The light sheet is generated with an Ultra CFR Nd:YAG laser system of Big Sky Laser. Each laser pulse contains $190 \mathrm{~mJ}$ at a wavelength of $532 \mathrm{~nm}$. The sheet thickness was in the range of $0.5 \mathrm{~mm}$. Perpendicular to the laser sheet, a PCO 1600 camera system by PCO AG was set up at a distance of about $200 \mathrm{~mm}$ for the acquisition of the particle images. The LabSmith timing unit by LC880 controls the trigger pulses for both components with an accuracy of $100 \mathrm{ps}$. The camera was equipped with the MakroPlanar 2/35 ZF lens by Carl Zeiss AG. The FOV to capture a global view of the wake resolves about $134 \times 100 \mathrm{~mm}^{2}$. To accommodate for the base geometry adaptation, the FOVs are equally shifted downstream with increasing base adapter lengths. Due to the high depth of focus, the choice for the aperture setting has turned out to be unfortunate. The wind tunnel nozzle is faintly visible in the background of the raw images for occasionally weak seeding. For this reason, results are not discussed in that flawed range, meaning if they are located upstream from $x / D<0.15$ and related to the incoming freestream $(r / D>0.5)$. The FOV for the relative nozzle length $L / D=1.2$ is depicted in Fig. 1, which also shows the coordinate system originating in the symmetry axis on the base. Seeding was accomplished with an in-house developed seeding generator providing titanium dioxide particles. Titanium dioxide of the type $K 1002$ from Kronos International, Inc. was used, which exhibits according to the manufacturer a number based average diameter and density of $d_{p}=0.23 \mu \mathrm{m}$ and $\rho_{p}=3800 \mathrm{kgm}^{-3}$, respectively. The particles were injected through an orifice into the flow upstream of position 'A' (Fig. 1). The jet was not seeded.

The analysis of the images was executed with PIVview V3.60 by PIVTEC GmbH. The relative motion between camera and wind tunnel model was measured for a close-up FOV to be $<40 \mu m$ (Saile 2019). For the current larger FOV, no motion could be detected between the raw images. Thus, it can be considered as negligible and was consequently not corrected. In total, a number of 345 images per run were evaluated with a window size of $32 \times 16$ px with an overlap of $4 \times 4 \mathrm{px}$. In physical units, one interrogation window without overlap has a size of $2.68 \times 1.34 \mathrm{~mm}^{2}$. The multigrid interrogation method with grid refinement was applied, the 'Whittaker' reconstruction (Raffel et al. 2007) was used for the sub-pixel peak fit and on the final pass, a B-spline interpolation scheme of $3^{\text {rd }}$ order was applied to cover the aspect of adaptive image deformation. The data were not interpolated.

An uncertainty analysis has been executed which is based on an approach suggested by Lazar et al. (2010) and Benedict and Gould (1996). The analysis takes into account the equipment-related uncertainty, the uncertainty due to the particle lag and the sampling uncertainty. In that order, the individual average contributions to the $95 \%$ confidence interval are found to be $\pm 0.24 \%, \pm 2.11 \%$, and $\pm 0.85 \%$ with respect to the incoming ambient flow velocity for the configuration with $L / D=0.45$, which featured the largest uncertainties in comparison to the other configurations. 'On average' relates to a box drawn in the near-wake downstream from the base between $0.15 \leq x / D \leq 1.2$ and $0.2 \leq r / D \leq 0.5$. In total, the 
individual contributions amount to an averaged uncertainty of $\pm 2.4 \%$. The averaged $95 \%$ confidence interval for the turbulent intensity components due to sampling errors was determined to be $< \pm 1 \%$. Further details are available in Saile (2019). Additionally, the results have been checked for peak-locking, which in consequence was ruled out, and with respect to the signal-to-noise ratio.

The methodological approach for the Proper Orthogonal Decomposition (POD) follows the traces of Wolf (2013), who refers Nguyen et al. (2010) for the governing equations. Both are using the method of snapshots or strobes as introduced by Sirovich (1987). In total, 345 instantaneous velocity snapshots were captured during an experiment and subsequently used for the POD analysis.

The underlying idea of the POD can be found in Eqn. 1, which is that the fluctuations of the velocity field $\boldsymbol{u}^{\prime}(\boldsymbol{x}, t)$ can be expressed as an expansion series of a time-varying scalar coefficient $\zeta_{k}(t)$ and a time-independent base function $\boldsymbol{\psi}_{k}(\boldsymbol{x})$. The base function $\psi_{k}(x)$ is equivalent to the $k^{\text {th }}$ eigenmode, and for the PIV results, can be interpreted as the $k^{\text {th }}$ flow field layer describing one of $K$ eigenmodes as the governing motions on top of the mean flow field $\overline{\boldsymbol{u}}(\boldsymbol{x})$. The number of modes $K$ corresponds to the number of time steps or snapshots $N$. Another output of the POD decomposition is the relative kinetic energy $\lambda_{k} / \sum \lambda_{k}$, which is used to rank the modes in descending order. The snapshot method (Sirovich 1987) yields at the computationally efficient determination of the corresponding POD basis functions $\psi$ and time-varying coefficients $\zeta$ for discrete experimental results such as given by PIV.

$\boldsymbol{u}(\boldsymbol{x}, t)=\overline{\boldsymbol{u}}(\boldsymbol{x})+\boldsymbol{u}^{\prime}(\boldsymbol{x}, t)=\overline{\boldsymbol{u}}(\boldsymbol{x})+\sum_{k=1}^{N} \zeta_{k}(t) \boldsymbol{\Psi}_{k}(\boldsymbol{x})$

VMK is equipped with a Z-type schlieren setup, which was used to capture the density gradients of the supersonic jet in the near-wake. The images were recorded with a Photron FASTCAM-APX RS camera, while the recording frequency was set to $17 \mathrm{kHz}$ with the shutter time at $5.0 e^{-5} \mathrm{~s}$. Each image is made up of $320 \times 240 \mathrm{px}$ and the magnification corresponds to $0.316 \mathrm{~mm} / \mathrm{px}$. The high recording frequency allowed the application of a spectral analysis to gain insights into the periodic behavior of the near-wake flow as shown later in context of Fig. 13. For the spectral analysis, the power spectral density was determined by using Welch's overlapped segment averaging estimator (Welch 1967). Each segment consisted of 2048 samples (with an overlap of 75\%) onto which a Hamming window was applied resulting in a minimal spectral resolution of $8.3 \mathrm{~Hz}$ up to a maximal resolution of $8.5 \mathrm{kHz}$.

\section{Results and discussion}

\subsection{Mean velocity distribution}

The contour plots shown in Fig. 3 reveal that the reattachment process undergoes a significant change with decreasing nozzle length. It is known from previous studies (Lê 2005; Saile et al. 2019a) that the shear layer reattachment moves downstream with increasing Mach numbers. For a nozzle length of $L / D=1.2$ and an ambient Mach number $\leq 0.7$, the reattachment type is solid. Then, at about Mach 0.8, the recirculation bubble features a common interface with the jet, meaning it becomes hybrid. The common interface grows larger if the nozzle length is subsequently decreased as done for the experiments at hand. As indicator for the reattachment location, one can use the stagnation streamline and the low speed corridor spreading up- and downstream from the vortex center (marked with a dashed arrow for the $L / D=1.2$-case).

For $L / D=1.2$, both indicators evidence a mean shear reattachment location along the solid nozzle wall. For 1.05, they both move closer to the tip of the nozzle, and finally for $L / D=0.9$, the mean reattachment location is evidenced right on the tip of the nozzle. Then, for $L / D \leq 0.75$, an overhang of the mean recirculation bubble can be noticed. The mean axial flow in that region reverses while the stagnation streamline evolving from the shoulder of the base still anchors at the nozzle tip. In the area of jet influence, the ambient flow experiences a realignment and acceleration in the streamwise direction.

\subsection{Reynolds stress distribution}

\subsubsection{Axial turbulence intensity}

In Saile et al. (2019a), two highly turbulent regions were discussed. The first was attributed to the motion of the shear layer, which is commonly observed by all base flow investigations, and the second, to the 'dancing' motion of the large scale, clockwise rotating, coherent vortex. In the following description in the frame of Fig. 4, both regions are considered regarding the axial turbulence intensity content for the investigated nozzle lengths.

The remains of the 'dancing' vortex center are clearly visible (at $[(x, r) / D]=[0.57,0.31])$ along the nozzle surface. The excitation in this region persists for the various shorter nozzle lengths, but the graphs do not reveal a clear dependency with respect to the nozzle length. For instance, an isolated and distinct excitation is notable for $L / D=0.9$, but the distinctiveness disappears for the other nozzle lengths. There, the excitation from the shear layer is 


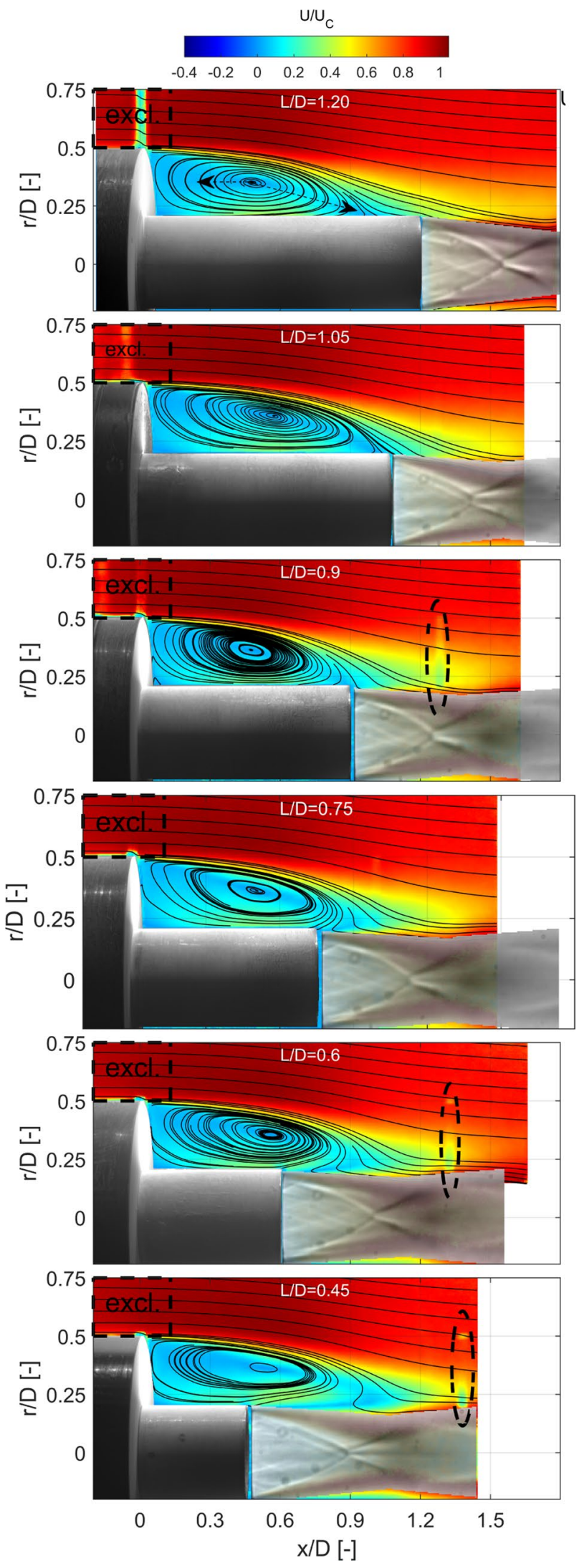

Fig. 3 Velocity distribution with streamlines for the relative nozzle lengths $L / D=1.2,1.05,0.9,0.75,0.6$ and 0.45 (from top to bottom). Disturbances due to laser light reflections are marked with a dashed box/ellipse
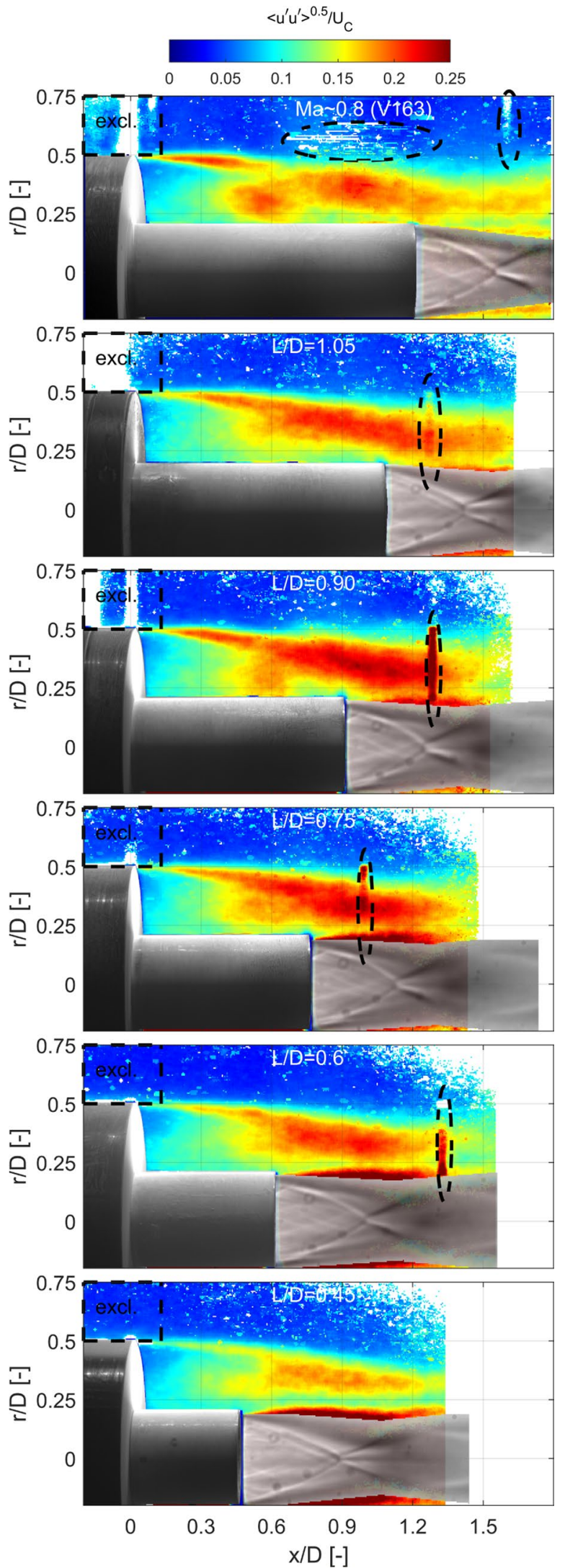

Fig. 4 Normalized axial turbulence intensity distribution for the relative nozzle lengths $L / D=1.2,1.05,0.9,0.75,0.6$ and 0.45 (from top to bottom). Disturbances due to laser light reflections are marked with a dashed box/ellipse 
Fig. 5 Normalized Reynolds stress distribution for the relative nozzle lengths $L / D=1.2,1.05,0.9,0.75,0.6$ and 0.45 (from top to bottom). The crosses ' $x$ ' mark the locations of the Reynolds shear stress extraction for Fig. 6. Disturbances due to laser light reflections are marked with a dashed box/ellipse

superimposed with the 'dancing' vortex center excitation, which obstructs a clear isolation of the two influences.

Generally, it can be stated that the shear layer excitation dominates. It increases with shorter nozzles, reaches a maximum for $L / D=0.9$ and decreases again for even shorter nozzles. The evolution here points at the existence of a configuration which is most unfavorable regarding shear layer oscillations. This is a discussion point pursued in the coming sections.

\subsubsection{Reynolds shear stress}

The Reynolds shear stress distribution as function of the relative nozzle length is presented Fig. 5. As before, the absolute level steadily increases up to a nozzle length $L / D=0.9$, decreases from there for larger nozzle lengths before seemingly reaching a plateau. To quantify this behavior, the value at the 'center of gravity' of the 'island' with a Reynolds shear stress level above $95 \%$ of the maximum value is extracted and listed in Table. 2. As further observation it can be stated that the effect of a 'dancing' main vortex center is less pronounced in the Reynolds shear stress distribution. Previous data presented in Saile et al. (2019a) already indicated that it predominantly affects the axial velocity fluctuations.

\subsection{Reynolds stress related to the nozzle length}

The previous trends regarding the turbulence intensity and Reynolds shear stress are now plotted in Fig. 6 as function of the relative nozzle length. The level for both is extracted from the point determined as maximum shear layer excitation (Table 2). The error bars are according to the 95\% confidence interval as suggested by Benedict and Gould (1996) for the sampling uncertainty.

Figure 6 now depicts clearly the increase in maximum Reynolds shear stress level and the turbulence intensity location up to a nozzle length of 0.9 . A further nozzle length increase seems to lead to a plateau at a lower turbulence level. This observation correlates with the finding that the excited area appears to shrink from a nozzle length of $L / D=1.05$ to 1.2 (Fig. 5). In other words, it seems like the influence of the jet on the reattachment process decreases and reaches a plateau if the exhaust jet is placed at a location farther downstream.

Moreover, the same tendency can also be found in the results by van Gent et al. (2017b). There, the fluctuations
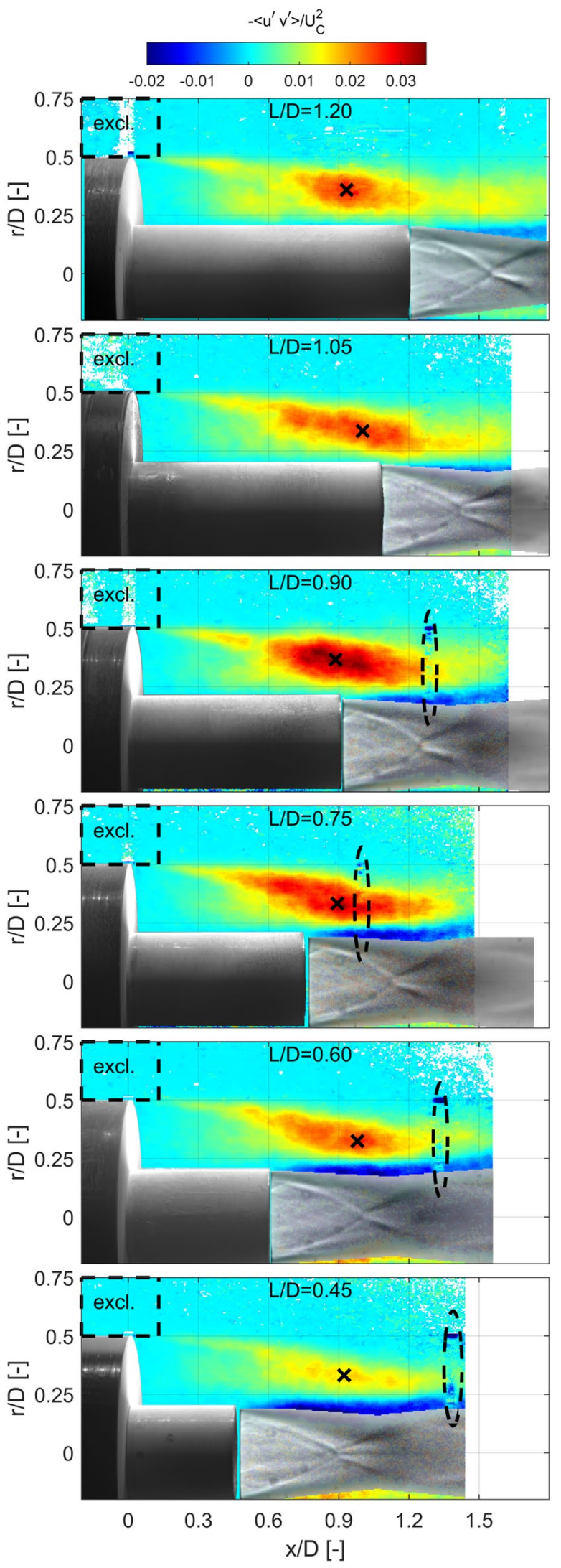
Table 2 The magnitude of the minimum Reynolds shear stress level $\left(u^{\prime} v^{\prime}\right)_{\min } / U_{C}^{2}$, its corresponding position $[(x, r) / D]^{*}$ and the position of the vortex center $[(x, r) / D]_{v c}$

\begin{tabular}{llll}
\hline$L / D$ & {$[(x, r) / D]_{v c}$} & {$[(x, r) / D]^{*}$} & $\left(u^{\prime} v^{\prime}\right)_{\min } / U_{C}^{2}$ \\
{$[-]$} & {$[-]$} & {$\left[\mathrm{ms}^{-1}\right]$} & {$[-]$} \\
\hline 1.2 & {$[0.48,0.35]$} & {$[0.93,0.36]$} & -0.0259 \\
1.05 & {$[0.58,0.36]$} & {$[1.00,0.34]$} & -0.0249 \\
0.9 & {$[0.47,0.36]$} & {$[0.89,0.37]$} & -0.0345 \\
0.75 & {$[0.50,0.36]$} & {$[0.89,0.33]$} & -0.0304 \\
0.6 & {$[0.56,0.36]$} & {$[0.98,0.33]$} & -0.0252 \\
0.45 & {$[0.53,0.36]$} & {$[0.92,0.33]$} & -0.0138 \\
\hline
\end{tabular}

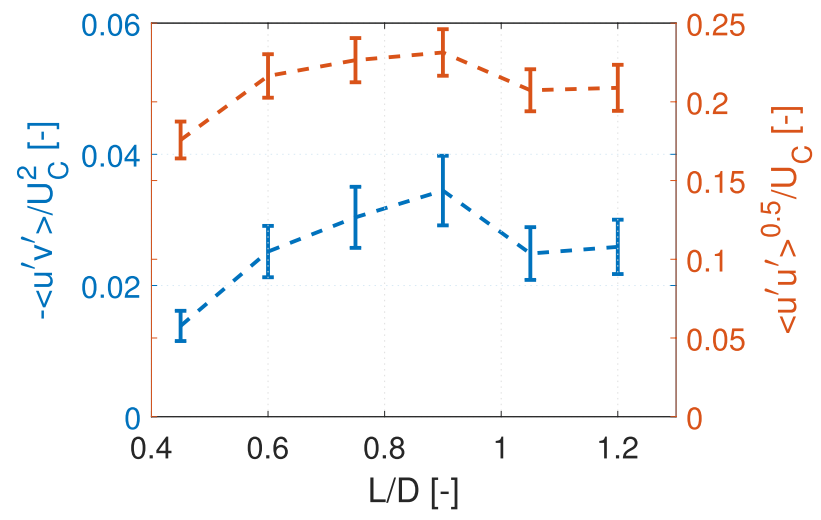

Fig. 6 The turbulence intensity and Reynolds shear stress level in dependence of the relative nozzle length

captured by the turbulent kinetic energy increase up to a nozzle length of $L / D=0.9$ and decrease again for the longer nozzle lengths $L / D=1.2$ and 1.8. For the latter two nozzles lengths, the shear layer reattaches on the mean at $L_{r} / D=1.1$, meaning the strongest excitation for that case is also reached if the configuration is as such that the mean shear layer reattachment takes place in the vicinity of the nozzle exit.

\subsection{Particularities of the amplified excitation}

\subsubsection{Probability density estimate}

The impingement location of the shear layer is assessed by means of the probability density estimate (based on a kernel smoothing density ksd function) of the axial velocity component just upstream from the nozzle exit. The corresponding function is shown in Fig. 7. Just upstream means $\Delta x / D=-0.01$ upstream from the individual nozzle exits at a radial distance of $r / D=0.225$. The corresponding point is named P1 and is depicted in the instantaneous velocity distribution plots below (Figs. 8, 9). In Fig. 7, the diamond

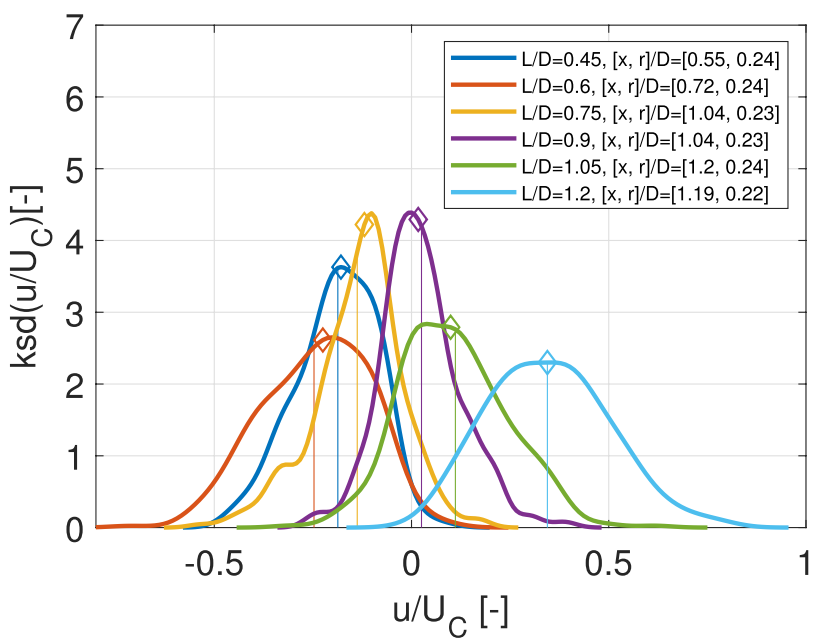

Fig. 7 Probability density estimate of the axial normalized velocity components sampled upstream from the nozzle exit at point P1. Diamond markers and thin continuous line denote the normalized mean and median axial velocity, respectively
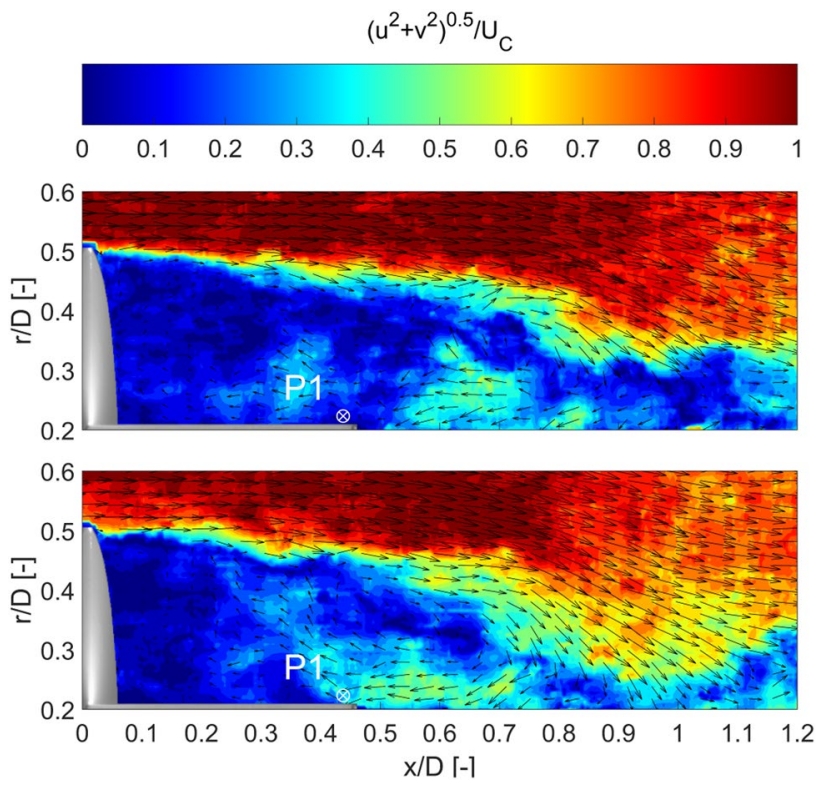

Fig. 8 Instantaneous velocity distribution for the relative nozzle length of $L / D=0.45$ with focus on point P1 just upstream from the nozzle exit. The top graph visualizes an example for a high axial velocity snapshot $\left(u / U_{C}=-0.01\right)$; the bottom graph a high reverse flow $\left(u / U_{C}=-0.31\right)$ snapshot. Every eighth and fourth vector is shown in $\mathrm{x}$ - and $\mathrm{r}$-direction, respectively

symbol and the thin line represent the mean and median value, respectively.

Downstream pointing axial flow components are an indicator that reattachment takes place on the solid nozzle surface, which is predominantly the case for $L / D=1.2$. For $L / D=1.05$, the axial flow components still point mostly in 


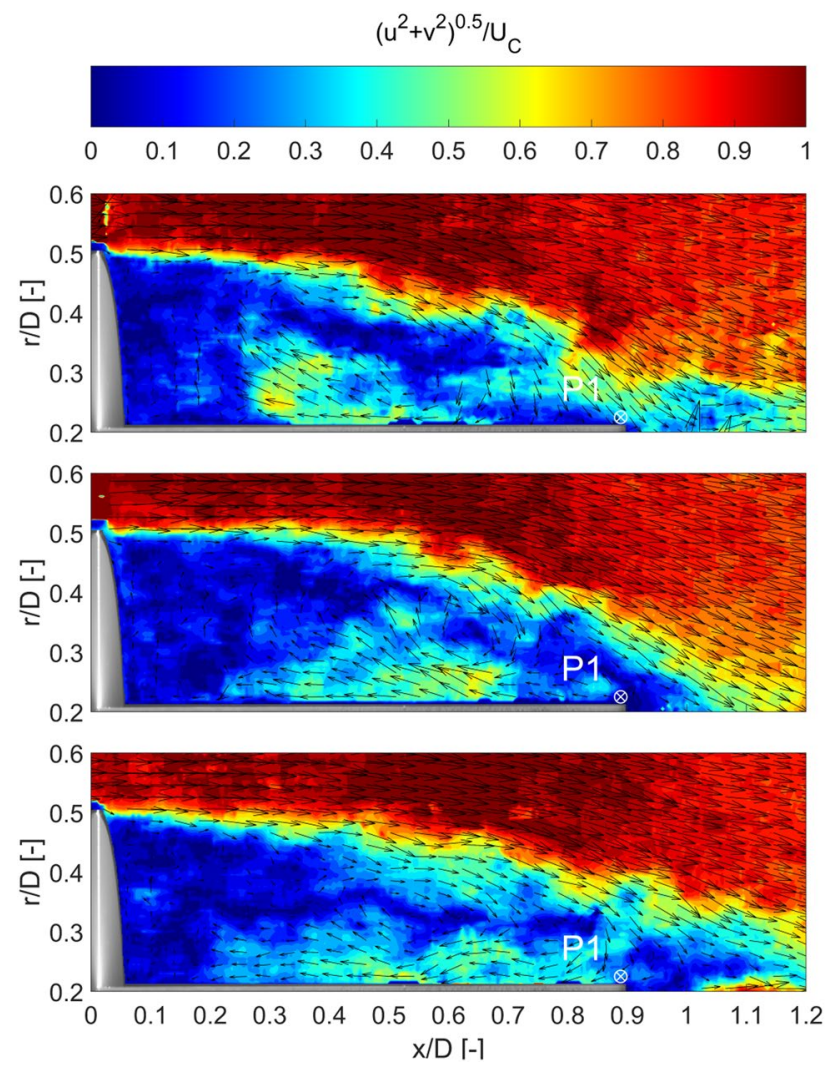

Fig. 9 Instantaneous velocity distribution for the relative nozzle length of $L / D=0.9$ to visualize a positive $\left(u / U_{C}=0.22\right)$, close to zero $(0.04)$ and negative axial velocity snapshot $(-0.23)$ at point P1 just upstream from the nozzle exit. Every eighth and fourth vector is shown in $\mathrm{x}$ - and $\mathrm{r}$-direction, respectively

the downstream direction, but a significant share also points in the opposite direction. Then, for $L / D=0.9$, the median shows that the ratio between upstream and downstream pointing axial velocity components is about equal. The tendency of diminishing downstream pointing flow components continues for the lower nozzle lengths until for $L / D=0.6$ they become insignificant. In other words, the shear layer reattachment is presumably purely fluidic for $L / D \leq 0.6$. To conclude, the lowest excitations are found if the shear layer either completely reattaches on the nozzle or on the jet. Vice versa, the strongest excitation is found if the shear layer reattaches on average at the tip of the nozzle.

\subsubsection{Instantaneous velocity distribution}

The nature of shear layer reattachment seems to play an important role with respect to the intensity of fluctuations or Reynolds stresses. To assess the specifics, representative instantaneous velocity distributions of a fluidic, hybrid and solid reattachment configuration are opposed in the following. The fluidic and hybrid type is represented by instant snapshots of the configuration with a nozzle length of
$L / D=0.45$ (Fig. 8) and 0.9 (Fig. 9), respectively. Snapshots of the configuration with $L / D=1.2$ as the representative for the solid reattachment were already shown in Saile et al. (2019a) (there, it was shown with the purpose to identify the drive behind high turbulence regions). The snapshots are chosen as such to reflect clear opposites in the spectrum of possible velocities at the point just upstream of the nozzle exit $\mathrm{P} 1$ as given in Fig. 7.

For the solid reattachment case (Saile et al. 2019a), the recirculation bubble is completely enclosed between the walls and the large-scale vortex completely realigns on its impingement upstream and downstream along the nozzle wall without being significantly influenced by the jet.

In contrast thereto, the recirculation bubble is not enclosed by the base and nozzle wall, but always exposed to the jet environment independent of the extent of the shear layer motion for the configuration with $L / D=0.45$. This can be seen in Fig. 8 while the top and bottom graphs depict an exemplary low and high axial velocity snapshot $\left(u / U_{C}=-0.31\right.$ and -0.01$)$. Despite the observation that the large scale features do not seem to be altered fundamentally from what was observed for $L / D=1.2$ (Saile et al. 2019a), meaning that the large-scale, clockwise rotating vortex is still present and that this vortex still provides a mass influx due to the engulfment process, one can also detect differences. For the snapshot $u / U_{C}=-0.31$, the flow equally realigns along the jet. However, for the other snapshot, one can see that mass at the interface to the jet is directly entrained due to the jet. Farther downstream, mass appears to be entrained in the vicinity of $x / D=0.9$ for both cases. In other words, the entrainment due to the jet appears to limit the mass engulfment process into the recirculation region.

The high turbulence case with $L / D=0.9$ shown in Fig. 9 is in between the above described configurations. The graphs show three snapshots categorized by the direction and magnitude of the axial velocity just upstream from the nozzle exit. The axial velocity of the top, mid and bottom instance in time is positive $\left(u / U_{C}=0.22\right)$, close to zero (0.04) and negative $(-0.23)$, respectively. By means of the low speed corridor (dark blue) or the vectors, one can recognize that shear layer reattachment takes place on the solid nozzle wall (top), right at the tip of the nozzle (mid) and on the jet (bottom). A further contrast to the previous results concerns the backflow due to the large, clockwise rotating vortex: The backflow runs along the solid nozzle wall and is not exposed to ejection effects by the jet. To put it more simply, the backflow is 'protected' from being inhaled and ejected by the jet.

\subsection{Proper orthogonal decomposition}

A proper orthogonal decomposition (POD) was applied on the instantaneous velocity fields to extract statistically dominant structures based on the turbulent kinetic energy 


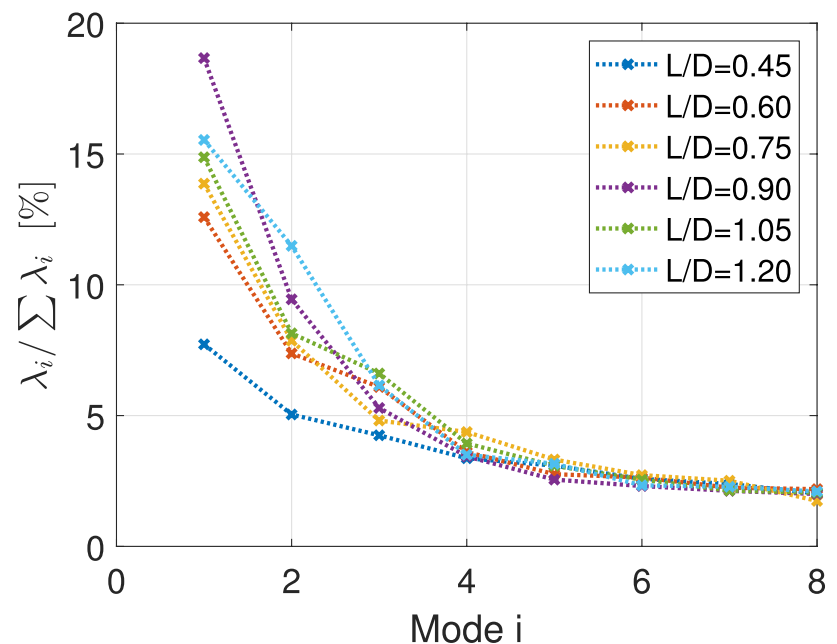

Fig. 10 POD energy spectra of the supersonic exhaust jet configurations with the relative nozzle lengths $L / D$ ranging from 0.45 to 1.2 for an ambient Mach number of 0.8

share. The corresponding POD energy spectra are shown in Fig. 10. It can be seen that the POD energy shifts in accordance with the modifications to the base geometry as already noted in context of the turbulence intensity in Fig. 6. For the first POD mode, an increase in the relative nozzle length concurs with an energy increase up to $19 \%$ for $L / D=0.9$ before reaching a steady level in the range of $15 \%$ for larger nozzles $(L / D \geq 1.05)$. The second mode also takes over a substantial share, which is in the range of half of the first mode. Generally, the first ten modes capture between $34 \%$ $(L / D=0.45)$ to $50 \%(L / D=1.2)$ of the total kinetic energy share, while the first two modes are responsible for close to 20 to $30 \%$ of the share. For this reason, these two modes are presented in the following.

The first and second POD velocity modes of the various base configurations are shown in Figs. 11 and 12, respectively. The first observation concerns the influence of nozzle length on the coherent structures and it can be stated for both modes that - apart from the magnitude of the excitation - its impact appears to be small: the first POD velocity modes are generally comparable with respect to the excitation location and the same is valid for the second POD velocity modes. In other words, the nozzle length or the exposure to the jet has no significant influence on the nature of the energetically most dominant near-wake flow dynamic features, but on its magnitude.

Second, both modes show coherent structures, which correlate with respect to the scale and the location with the large, clockwise-rotating vortex and the shear layer. Thus, these two modes appear to capture significant contributions of the dynamic behavior of the most dominant features. It can be seen that the first mode mainly imposes an overall

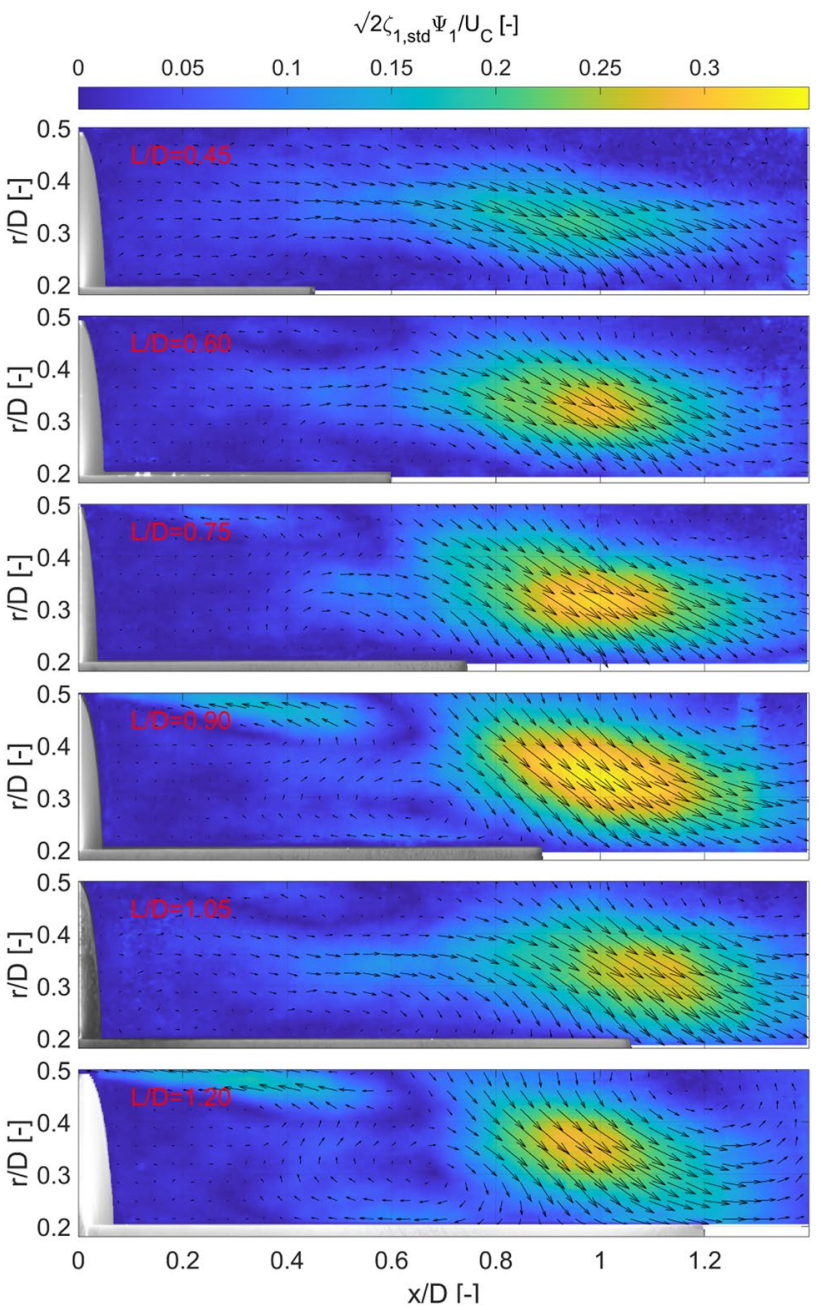

Fig. 11 First POD velocity modes of the supersonic exhaust jet configurations with the relative nozzle lengths $L / D$ ranging from 0.45 to 1.2 for an ambient Mach number of 0.8

oscillation of the shear layer causing a growth and decay motion of the recirculation bubble. The second mode features counteracting coherent structures in the shear layer region, meaning it introduces a swinging motion to the shear layer dynamics.

\subsection{Spectral analysis}

Apart from the flow topology of the jet, the schlieren recordings also provide insights into the dominant frequencies in the near-wake due to the high recording rate of the camera, which, in consequence, enabled the application of a spectral analysis. For that, the spectra of each pixel of the high-speed schlieren recordings were evaluated and, in a collecting step, averaged. In other words, the result is essentially the averaged spectra of the gradient density in the near-wake field. 


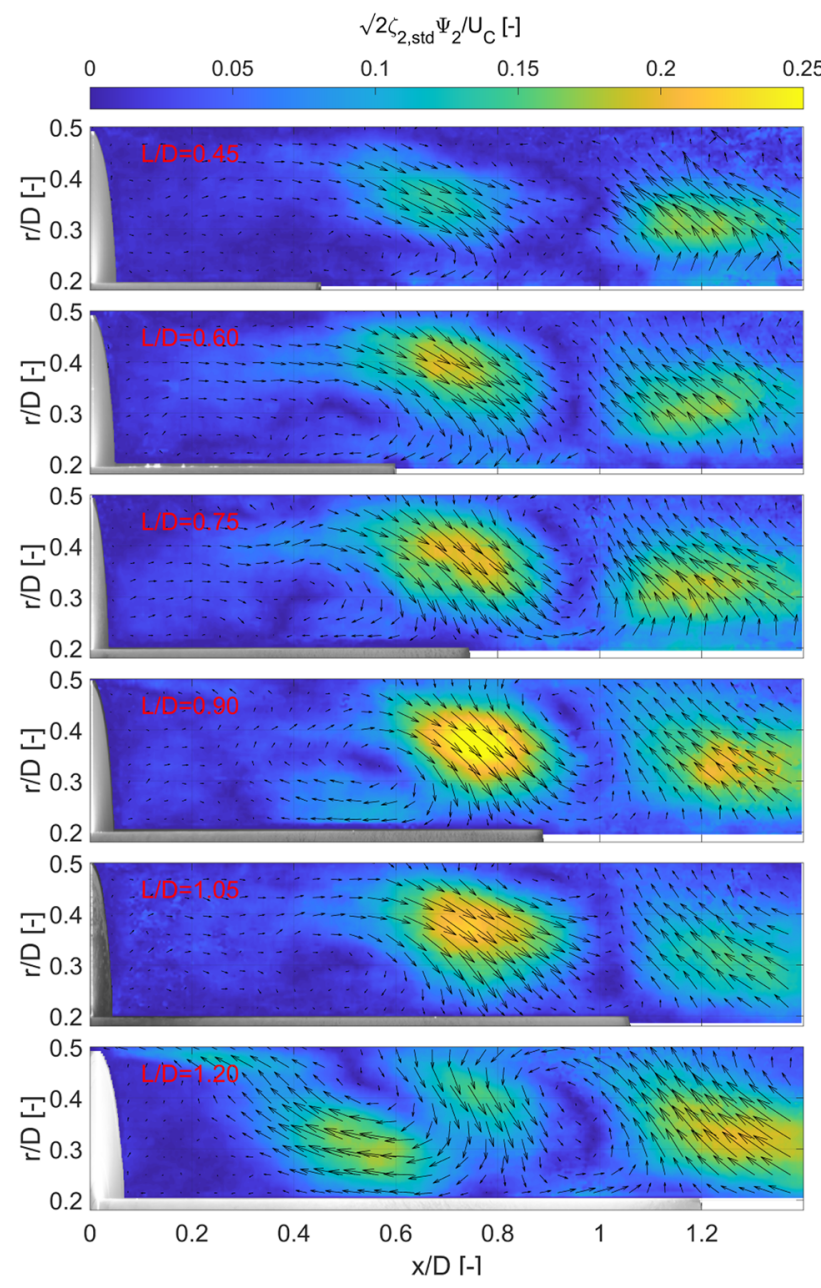

Fig. 12 Second POD velocity modes of the supersonic exhaust jet configurations with the relative nozzle lengths $L / D$ ranging from 0.45 to 1.2 at an ambient Mach number of 0.8

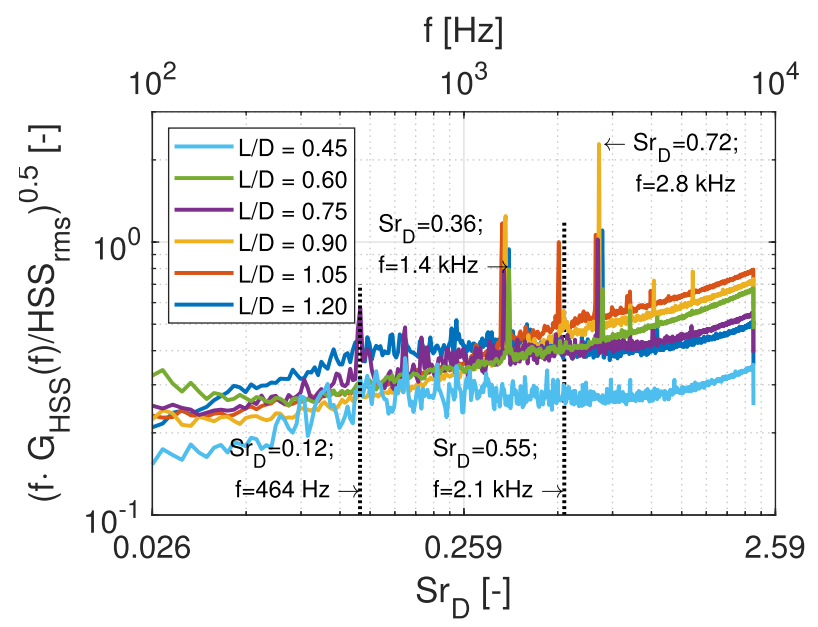

Fig. 13 High-speed schlieren spectra as function of the Strouhal number $S r_{D}$ and frequency $f$ for the jet:on-case and various relative nozzle lengths $L / D$ at Mach 0.8
The results are shown in Fig. 13 in terms of the nondimensional frequency $S r_{D}$ and the pre-multiplied power spectral density $\left.\left(f . G_{\mathrm{HSS}}(f)\right) / H S S_{\mathrm{rms}}\right)^{0.5}$. Note that nondimensional frequency $S r_{D}$ is defined as $S r_{D}=f \cdot D / U_{C}$, meaning it is based on the diameter $D$ of the main body and the measured frequency $f$ normalized with the ambient velocity $U_{C}$. Figure 13 reveals that the most dominant periodic oscillation in the near-wake can be found at $S r_{D}=0.36$ and higher harmonics for all nozzle lengths except for $L / D=0.45$.

On the one hand, the independence of the frequency to the nozzle length is consistent with the observation that the dominant POD modes in the near-wake do not change due to the applied variation. This in turn agrees with the splitter plate experiments by Roshko (1955), which revealed that the near-wake flow frequency depends on the reattachment location of the large, clockwise-rotating vortex. Applied to the case at hand: the nature of the near-wake flow features do not change, thus the associated frequencies remain constant as well. On the other hand, the near-wake flow is typically found to oscillate with a dominant Strouhal number of $S r_{D} \approx 0.2$ in that flow regime, which is attributed to the mutual interaction between the separated shear layers. However, as shown by Deprés et al. (2004), the presence of a jet or a solid body/nozzle such as given for the case at hand suppresses this asymmetric interaction mechanism restricting possible oscillations to symmetric, higher-order modes such as associated with the swinging of the shear layer at $S r_{D}=0.36$ (Statnikov et al. 2017).

The exception for the $L / D=0.45$ configuration can presumably be explained with the observation from the POD results above, which is that the excitation mechanism is comparably weak for that case, meaning the signal captured by the schlieren measurements is equally weak, and thus, not detected. The relative weakness in that must be considered in context with Ch. 3.4.1 where it is shown that for $L / D=0.45$, the shear layer reattaches exclusively on the jet (see Figs. 3,7 ) and the jet entrains the mass coming from the large, clockwise-rotating vortex (Fig. 8). This indicates that the vortex shedding process does not take place along the solid surface of the nozzle, but instead, it is dampened due to entrainment effect induced by the jet.

\subsection{Summarizing discussion on the near-wake flows of various base geometries}

The previous study (Saile et al. 2019a) attributed the high turbulent intensity patches in the contour plot to either the 'dancing' vortex center of the large, clockwise-rotating vortex or to the shear layer motion. The current results also feature these intensified areas (Fig. 4) and due to the similarities to the previous results it seems safe to assume 
that the origin of the excitation sources remain the same. The question now is: What are the special conditions for the unwanted increased turbulent intensity region for the relative nozzle length of 0.9 (Fig. 6)?

The probability density function of the velocity samples just upstream of the nozzle exit (Fig. 7) reveal that the $L / D=0.9$-case represents the configuration for which shear layer reattachment on the solid nozzle wall is equally frequent as on the exhaust jet. On the mean, the flow directly reattaches on the nozzle tip, meaning without flow reversal as for $L / D<0.9$ and without impinging on the solid nozzle wall before as for $>0.9$.

It appears that two criteria must be given for a maximal excitation: First (criterion 1), the mean recirculation bubble must be frequently connected with the jet, which corresponds the hybrid shear layer reattachment type. Under these circumstances, the results indicate that some dynamic exchange process is taking place, which in turn feeds turbulent excitations in the recirculation bubble. A symptom of which seems to be the 'dancing' main vortex center. Statnikov et al. (2017) suggested a 'cross-pumping' motion where a vortical structure is shed from the main vortex every time the separation bubble is close to the nozzle exit. In fact, the POD of the current data set suggests as dominant POD mode such a vortex shedding process (Saile 2019) for the Mach 0.8-case. Such a vortex shedding process essentially equals a mass loss from the base region. Additionally, the jet is known to eject mass from the base region due to entrainment or base suction (e.g., Schoones and Bannink (1998), Deprés (2003), Wolf (2013)).

However, the turbulent excitation does not increase continuously with an increasing interface to the jet (or decreasing nozzle lengths). Consequently, there must be a second aspect, which introduces a dampening effect. The instantaneous velocity distributions for the relative nozzle length $L / D=0.45$ (Fig. 8) show that the excitation decrease correlates with the increased mass flow ejection from the large, clockwise-rotating vortex. For the larger nozzle length $L / D=0.9$ on the other hand (Fig. 9), the backflow induced by the vortex travels unhindered upstream along the solid nozzle wall. Thus, it appears that the excitation becomes maximal if the large, clockwise-rotating vortex impinges on the nozzle wall only such that the complete energy and mass of that engulfing vortex is transferred to recirculation bubble. The description of the latter forms the second criterion (criterion 2).

It is hypothesized now that the $L / D=0.9$ configuration at Mach 0.8 poses a situation for which the vortex-shedding driven entrainment by the jet and mass supply by the engulfing large, clockwise-rotating vortex is maximal. Obviously, the design goal for a space launcher is the opposite: the minimization of dynamic processes and inherent base pressure fluctuations. Thus, the occurrence of such a circumstance should be avoided.

The plateau regarding the turbulent quantities for nozzle lengths larger than $L / D=1.05$ (Fig. 6) is addressed as last point. It appears reasonable that a plateau is reached if the reattachment predominantly takes place on the solid nozzle wall as it can be found for these nozzle lengths $\geq 1.05$ (Fig. 7). Then the reattachment conditions are less and less influenced by the downstream environment.

\section{Conclusions and outlook}

The research questions of the current study posed above suggest the following answers: Yes, the excitation in the near-wake is dependent on the nozzle length, and yes, a most unfavorable base geometry appears to exist. For the investigated configurations, it can be found for a relative nozzle length of $L / D=0.9$. Then, upstream pointing velocity fluctuations are as frequent as downstream pointing fluctuations. It further corresponds to the configuration for which the reattachment on the mean does not take place along the solid nozzle wall, but just at the tip of the nozzle (without overhang). It was further suggested that an engulfmententrainment process driven by a large-scale vortex and the jet is the driver for the relatively strong fluctuations. This is supported by the finding that the predominant oscillation takes place with a Strouhal number of 0.36 , which is associated with the interrelated near-wake dynamics called shear layer swinging. Consequently, it appears recommendable to avoid such a configuration due to its presumable impact as loads on the base structures of the space launcher.

Acknowledgements Financial support has been provided by the German Research Foundation (Deutsche Forschungsgemeinschaft - DFG) in the framework of the Sonderforschungsbereich Transregio 40. Further, thanks a lot to the colleagues and the staff for their support!

Open Access This article is licensed under a Creative Commons Attribution 4.0 International License, which permits use, sharing, adaptation, distribution and reproduction in any medium or format, as long as you give appropriate credit to the original author(s) and the source, provide a link to the Creative Commons licence, and indicate if changes were made. The images or other third party material in this article are included in the article's Creative Commons licence, unless indicated otherwise in a credit line to the material. If material is not included in the article's Creative Commons licence and your intended use is not permitted by statutory regulation or exceeds the permitted use, you will need to obtain permission directly from the copyright holder. To view a copy of this licence, visit http://creativecommons.org/licenses/by/4.0/. 


\section{References}

Benedict L, Gould R (1996) Towards better uncertainty estimates for turbulence statistics. Exp Fluids 22(2):129-136. https://doi.org/ $10.1007 / \mathrm{s} 003480050030$

David S, Radulovic S (2005) Prediction of buffet loads on the Ariane 5 afterbody. In: 6th symposium on launcher technologies, Munich, Germany, 8-11 November 2005, pp 1-12

Deck S, Thorigny P (2007) Unsteadiness of an axisymmetric separating-reattaching flow: numerical investigation. Phys Fluids 19(6):1-20. https://doi.org/10.1063/1.2734996

Deprés D (2003) Analyse physique et modélisation des instationnarits dans les Écoulements d'Arrière-Corps Transoniques. $\mathrm{PhD}$ thesis, Université de la Méditerranée Aix-Marseille II

Deprés D, Reijasse P, Dussauge J (2004) Analysis of unsteadiness in afterbody transonic flows. AIAA J 42(12):2541-2550. https://doi. org/10.2514/1.7000

DLR (2019) Vertical test section cologne (VMK), Supersonic and Hypersonic Technology Department. http://www.dlr.de/as/en/ desktopdefault.aspx/tabid-194/407_read-5445/

Fuchs H, Mercker E, Michel U (1979) Large-scale coherent structures in the wake of axisymmetric bodies. J Fluid Mech 93(01):185207. https://doi.org/10.1017/S0022112079001841

Goethert B, Barnes L (1960) Some studies of the flow pattern at the base of missiles with rocket exhaust jets. Tech. rep, DTIC Document

Hannemann K, Ludeke H, Pallegoix JF, Ollivier A, Lambare H, Maseland JEJ, Geurts EGM, Frey M, Deck S, Schrijer FFJ, Scarano F, Schwane R (2011) Launch vehicle base buffeting-recent experimental and numerical investigations. In: Ouwehand L (ed) 7th European Symposium on Aerothermodynamics, ESA Special Publication, vol 692, p 102

Lazar E, DeBlauw B, Glumac N, Dutton C, Elliott G (2010) A practical approach to PIV uncertainty analysis. In: 27th AIAA Aerodynamic Measurement Technology and Ground Testing Conference, 28 June 2010 - 01 July 2010, Chicago, Illinois, USA, p 4355, $10.2514 / 6.2010-4355$

Lê THH (2005) Étude expérimentale du Couplage entre l'Écoulement Transsonique d'Arrière-Corps et les Charges Latérales dans les Tuyères Propulsives. PhD thesis, Université de Poitiers, École Nationale Supérieure de Mécanique et d'Aérotechnique

Morkovin M (1964) Flow around circular cylinder - a kaleidoscope of challenging fluid phenomena. In Haswen, AG (Ed): Symposium of Fully Separated Flows pp 102-118

Nguyen TD, Wells JC, Mokhasi P, Rempfer D (2010) Proper orthogonal decomposition-based estimations of the flow field from particle image velocimetry wall-gradient measurements in the backward-facing step flow. Measurem Sci Technol 21(11):1-15. https://doi.org/10.1088/0957-0233/21/11/115406

Raffel M, Willert CE, Kompenhans J et al (2007) Particle image velocimetry: a practical guide. Springer, Berlin Heidelberg. https://doi. org/10.1007/978-3-540-72308-0

Roshko A (1955) On the wake and drag of bluff bodies. J Aeronautical Sci 22(2):124-132. https://doi.org/10.2514/8.3286

Roshko A, Lau JC (1965) Some observations on transition and reattachment of a free shear layer in incompressible flow. Proc 1965 Heat Transfer and Fluid Mechanics Institute, edited by AF Charwat, Stanford University Press, Stanford pp 157-167

Saile D (2019) Experimental analysis on near-wake flows of space transportation systems. PhD thesis, Rheinisch-Westflische Technische Hochschule Aachen

Saile D, Kirchheck D, Gülhan A, Banuti D (2015) Design of a hot plume interaction facility at DLR Cologne. In: Proceedings of the 8th European Symposium on Aerothermodynamics for Space Vehicles, 02 Mar 2015 - 06 Mar 2015, Lisbon, Portugal, pp 1-17
Saile D, Kühl V, Gülhan A (2019a) On the subsonic near-wake of a space launcher configuration with exhaust jet. Exp Fluids 60(11):165. https://doi.org/10.1007/s00348-019-2801-7

Saile D, Kühl V, Gülhan A (2019b) On the subsonic near-wake of a space launcher configuration without jet. Exp Fluids 60(4):50. https://doi.org/10.1007/s00348-019-2690-9

Saile, D, Gülhan, A, Henckels, A, Glatzer, C, Statnikov, V, Meinke, M (2013) Investigations on the turbulent wake of a generic space launcher geometry in the hypersonic flow regime. In: Knight D, Lipatov I, Reijasse P (eds) EUCASS Proceedings Series - Advances in AeroSpace Sciences, EDP Sciences, vol 5, pp 209-234, 10.1051/eucass/201305209, https://doi.org/10. 1051/eucass/201305209

Scharnowski S (2013) Investigation of turbulent shear flows with high resolution PIV methods. PhD thesis, Universität der Bundeswehr München, Fakultät für Luft- und Raumfahrttechnik

Scharnowski S, Statnikov V, Meinke M, Schröder W, Kähler CJ (2015) Combined experimental and numerical investigation of a transonic space launcher wake. In: Knight D, Bondar Y, Lipatov I, Reijasse P (eds) EUCASS Proceedings Series Advances in AeroSpace Sciences, vol 7, pp 311-328, 10.1051/ eucass/201507311, https://doi.org/10.1051/eucass/201507311

Scharnowski S, Bolgar I, Kähler C (2016) Interaction of a generic space launcher wake with a jet plume in sub-, trans- and supersonic conditions. NIOPLEX International Workshop on NonIntrusive Optical Flow Diagnostics, 25-26 October 2016, Delft, Netherlands

Schoones M, Bannink W (1998) Base flow and exhaust plume interaction. Part 1: Experimental study. Series 01: Aerodynamics 15, Delft University Press, http://resolver.tudelft.nl/uuid:ad12e d62-1869-4daa-86b1-ea2785163a1d

Schrijer F, Sciacchitano A, Scarrano F, Hannemann K, Pallegoix JF, Maseand J, Schwane R (2011) Experimental investigation of base flow buffeting on the Ariane 5 launcher using high speed PIV. In: 7th European Symposium on Aerothermodynamics, 9-12 May 2011, Brugge, Belgium, vol 692. p 103

Schrijer F, Sciacchitano A, Scarano F (2014) Spatio-temporal and modal analysis of unsteady fluctuations in a high-subsonic base flow. Phys Fluids 26(8):1-19. https://doi.org/10.1063/1.4891257

Schwane R (2015) Numerical prediction and experimental validation of unsteady loads on Ariane 5 and Vega. J Spacecraft Rockets 52(1):54-62. https://doi.org/10.2514/1.A32793

Sirovich L (1987) Turbulence and the dynamics of coherent structures: I. Coherent structures. Quart Appl Math 45(3):561-571. https://doi.org/10.1063/1.1693225

Statnikov V, Bolgar I, Scharnowski S, Meinke M, Khler C, Schrder W (2016) Analysis of characteristic wake flow modes on a generic transonic backward-facing step configuration. European J Mech - B/Fluids 59:124-134

Statnikov V, Meinke M, Schröder W (2017) Reduced-order analysis of buffet flow of space launchers. J Fluid Mech 815:1-25. https://doi.org/10.1017/jfm.2017.46

Triesch K, Krohn EO (1986) Die Vertikale Mestrecke der DFVLR in Köln-Porz (Stand 1986), DFVLR-Mitt. 86-22. Wissenschaftliches Berichtswesen der DFVLR, ISSN 0176-7739, Postfach 906058, 5000 Köln 90

van Gent P, Michaelis D, Van Oudheusden B, Weiss PE, de Kat R, Laskari A, Jeon YJ, David L, Schanz D, Huhn F et al (2017a) Comparative assessment of pressure field reconstructions from particle image velocimetry measurements and lagrangian particle tracking. Exp Fluids 58(4):33. https://doi.org/10.1007/ s00348-017-2324-Z

van Gent P, Payanda Q, Brust S, van Oudheusden B, Schrijer F (2017b) Experimental study of the effects of exhaust plume and nozzle length on transonic and supersonic axisymmetric base flows. 
7th European Conference For Aeronautics And Space Sciences 0.13009/EUCASS2017-657

van Gent PL, Payanda Q, Brust SG, van Oudheusden BW, Schrijer FFJ (2019) Effects of exhaust plume and nozzle length on compressible base flows. AIAA J 57(3):1184-1199. https://doi.org/ 10.2514/1.J057314

Weiss PE, Deck S (2013) Numerical investigation of the robustness of an axisymmetric separating/reattaching flow to an external perturbation using ZDES. Flow, Turbulence Combust 91:697715. https://doi.org/10.1007/s10494-013-9484-6

Weiss PE, Deck S, Robinet JC, Sagaut P (2009) On the dynamics of axisymmetric turbulent separating/reattaching flows. Phys Fluids 21(7):1-8. https://doi.org/10.1063/1.3177352

Welch P (1967) The use of fast fourier transform for the estimation of power spectra: a method based on time averaging over short, modified periodograms. IEEE Transac on Audio Electroacoustics 15(2):70-73. https://doi.org/10.1109/TAU.1967.1161901

\section{Authors and Affiliations}

\section{Dominik Saile $^{1}{ }^{1} \cdot$ Viktor Kühl $^{1} \cdot$ Ali Gülhan ${ }^{1}$}

Dominik Saile

dominik.saile@dlr.de

1 DLR German Aerospace Center, Institute of Aerodynamics and Flow Technology, Supersonic and Hypersonic
Westphal RV, Johnston J, Eaton J (1984) Experimental study of flow reattachment in a single-sided sudden expansion. NASA Contr Rep 3765:1-284

Wolf CC (2013) The subsonic near-wake of bluff bodies. PhD thesis, Rheinisch-Westfälische Technische Hochschule Aachen, Fakultät für Maschinenwesen

Wong H, Meijer J, Schwane R (2007) Experimental and theoretical investigation of base-flow buffeting on Ariane5 launch vehicles. J Propul Power 23(1):116-122. https://doi.org/10.2514/1.19740

Publisher's Note Springer Nature remains neutral with regard to jurisdictional claims in published maps and institutional affiliations.

Technology Department, 51147 Linder Höhe, Cologne, Germany 